\title{
DISTRIBUTION SPATIALE ET MISE EN OEUVRE D'UNE POLITIQUE PUBLIQUE : LE CAS DE LA POLLUTION ATMOSPHERIQUE
}

\author{
Peter KNOEPFEL* \\ Corinne LARRUE*
}

\begin{abstract}
Résumé
Après avoir présenté l'arrangement politico-administratif retenu pour la mise en ceuvre de la politique de lutte contre la pollution de l'air en France, les auteurs constatent que les politiques mises en place dans trois régions prises à titre d'exemple diffèrent, tant sur le plan des instruments d'intervention retenus que sur les résultats obtenus; les facteurs explicatifs de ces différences sont alors analysés, et il apparaît que la distribution spatiale du problème de la pollution atmosphérique n'est pas le seul facteur explicatif des variations constatées entre les régions et entre les localités à l'intérieur des régions. Cette analyse conduit à une reflexion sur les effets distributifs de la mise en ceuvre de politiques publiques, qui ne se limitent pas aux seules politiques redistributives classiques.
\end{abstract}

\footnotetext{
* Institut de Hautes Etudes en Administration Publique (IDHEAP), Lausanne.
} 
La pollution atmosphérique par le $\mathrm{SO}_{2}$ (dioxyde de soufre) provient essentiellement des installations fixes de combustion, c'est-à-dire les installations de chauffage domestique (10\% des émissions de soufre), les centrales thermiques $(40 \%)$ et les foyers industriels : raffineries, sidérurgie essentiellement $(48 \%)(1)$. Cette pollution se mesure par le nombre de tonnes de soufre rejetées dans l'atmosphère (taux d'émission) ou la teneur de l'air en $\mathrm{SO}_{2}$ (taux d'immision). La pollution de l'air varie d'une région a l'autre : certains départements (comme le Nord, les Bouches-du-Rhône, la Seine Maritime) enregistrent de très fortes émissions ; et les taux de pollution des agglomérations de Paris, Marseille ou du Havre restent nettement plus élevés que ceux d'autres agglomérations. De plus, si globalement la qualité de l'air en France s'est améliorée durant les dix dernières années, cette évolution n'a pas été identique pour chacune des agglomérations. La pollution atmosphérique est donc un problème très localisé ; cet article, qui tente d'expliquer la politique de lutte contre ce type de pollution, pose la question des conséquences de la distribution spatiale de la pollution de l'air (distribution spatiale de la pression du problème), pour les politiques régionales de mise en cuvre, menées selon un programme identique pour l'ensemble du territoire.

Le programme de la politique de lutte contre la pollution atmosphérique et la marge d'appréciation des politiques de mise en œuvre
Comparé à des programmes similaires dans d'autres pays, le programme français qui établit et oriente la politique publique de lutte contre la pollution de l'air peut être caractérisé comme partiel (2). L'esquisse de la législation nationale, telle que présentée, met en évidence l'absence de plusieurs éléments importants dans le programme de contrôle du $\mathrm{SO}_{2}$. Malgré l'orientation de la législation vers le contrôle des immissions, ni la loi sur la pollution atmosphérique de 1961, ni les décrets d'application de 1963 et 1974, ni les arrétés ministériels pris en application de cette réglementation ne contiennent un objectif précis de qualité de l'air à atteindre ; cette absence est volontaire car il incombe aux politiques régionales de définir ces objectifs en fonction de ce qu'elles estiment raisonnable à atteindre. Contrairement à ce qu'on trouve dans d'autres pays (3), la législation française ne contient pas non plus de standards d'émission, qui limiteraient d'une façon générale les émissions des installations de combustion. Les exigences générales formulées dans une vingtaine de circulaires sont très abstraites, ce qui laisse une marge d'appréciation considérable aux autorités chargées de leur application. II en est de même pour les éléments substantiels qui fixent les régimes de la Zone de Protection Spéciale et de la Zone d'Alerte.

Le tableau suivant caractérise les trois instruments d'intervention que la législation française met à disposition. 


\begin{tabular}{|c|c|c|c|}
\hline CARACTERISTIQUES & $\begin{array}{c}\text { CONTRAINTES } \\
\text { INDIVIDUELLES } \\
\text { LES INSTALLATIONS } \\
\text { CLASSEES }\end{array}$ & $\begin{array}{c}\text { CONTRAINTES } \\
\text { SEMI-COLLECTIVES } \\
\text { LA ZONE D'ALERTE } \\
\text { (ZA) }\end{array}$ & $\begin{array}{c}\text { CONTRAINTES } \\
\text { COLLECTIVES } \\
\text { LA ZONE DE PROTEC- } \\
\text { TECTION SPECIALE }\end{array}$ \\
\hline BASE LEGALE & $\begin{array}{l}\text { Législation des Installa- } \\
\text { tions Classées (Loi de } \\
1917 \text { transformée en } \\
1976 \text {; Rubrique n } 153 \\
\text { bis de la nomenclature } \\
\text { et env. } 20 \text { circulaires) }\end{array}$ & $\begin{array}{l}\text { Législation sur la pollu. } \\
\text { tion atmosphérique } \\
\text { (Décret de 1974) }\end{array}$ & $\begin{array}{l}\text { Législation sur la pollu. } \\
\text { tion atmosphérique } \\
\text { (Loi de } 1961 \text {, Décret de } \\
1963 \text { transformé en } \\
1974 \text { ) }\end{array}$ \\
\hline $\begin{array}{l}\text { ORIENTATION } \\
\text { (EMISSIONS/ } \\
\text { IMMISSIONS) }\end{array}$ & $\begin{array}{l}\text { Dans la pratique cet ins- } \\
\text { trument peut être utilisé } \\
\text { selon les deux orienta- } \\
\text { tions, par manque de } \\
\text { définition }\end{array}$ & $\begin{array}{l}\text { Immissions (éviter les } \\
\text { pointes de pollution par } \\
\text { une procédure d'alerte } \\
\text { en cas de conditions mé- } \\
\text { téorologiques défavo. } \\
\text { rables) }\end{array}$ & $\begin{array}{l}\text { Immissions (avec effet } \\
\text { important sur les émis. } \\
\text { sions) }\end{array}$ \\
\hline $\begin{array}{l}\text { INSTRUMENT } \\
\text { JURIDIQUE DE } \\
\text { L'INTERVENTION }\end{array}$ & $\begin{array}{l}\text { Autorisation (unités im- } \\
\text { portantes) ou Déclaration } \\
\text { (unités moyennes) déli- } \\
\text { vrées avec prescriptions } \\
\text { individuelles sous forme } \\
\text { d'arrêté préfectoral }\end{array}$ & $\begin{array}{l}\text { Obligation pour les gros- } \\
\text { ses unités de combustion } \\
\text { de changer de combusti- } \\
\text { ble ou de réduire leur } \\
\text { activité lorsqu'un certain } \\
\text { seuil de pollution est } \\
\text { atteint }\end{array}$ & $\begin{array}{l}\text { Interdiction d'utiliser } \\
\text { un combustible trop char- } \\
\text { gé en soufre et fixation } \\
\text { de la teneur maximale } \\
\text { en soufre des combusti- } \\
\text { bles autorisés dans une } \\
\text { zone délimitée }\end{array}$ \\
\hline $\begin{array}{l}\text { DIMENSIONS } \\
\text { PRINCIPALES DE } \\
\text { L'INTERVENTION }\end{array}$ & $\begin{array}{l}\text { Normes de rejet de pol. } \\
\text { luants (limites indivi- } \\
\text { duelles d'émission) ou } \\
\text { hauteur minimale des } \\
\text { cheminées }\end{array}$ & $\begin{array}{l}\text { Taux de soufre des fueis } \\
\text { ou activités de combus- } \\
\text { tion }\end{array}$ & $\begin{array}{l}\text { Taux de soufre des com- } \\
\text { bustibles et notamment } \\
\text { des fuels-oils domesti- } \\
\text { ques }\end{array}$ \\
\hline $\begin{array}{l}\text { ADMINISTRATION } \\
\text { COMPETENTE POUR } \\
\text { INTERVENIR (dans } \\
\text { la pratique) }\end{array}$ & $\begin{array}{l}\text { La DII fait une recom- } \\
\text { mandation au Préfet, } \\
\text { après consultation de la } \\
\text { DDASS, DDE, DDA et } \\
\text { du Conseil Général }\end{array}$ & $\begin{array}{l}\text { La DII propose le conte- } \\
\text { nu de l'arrêté préfectoral } \\
\text { qui indique les entrepri. } \\
\text { ses incluses et les moda. } \\
\text { tés de l'alerte }\end{array}$ & $\begin{array}{l}\text { Forte implication du ni- } \\
\text { veau national (arrêtés } \\
\text { interministériels, Environ. } \\
\text { nement, Industrie, Santé, } \\
\text { et intérieur) }\end{array}$ \\
\hline $\begin{array}{l}\text { UTILISATION DE } \\
\text { L'INSTRUMENT }\end{array}$ & Dans toutes les régions & $\begin{array}{l}14 \text { zones d'alertes créées } \\
\text { ou en cours de création } \\
\text { depuis } 1974 \text { (dont Dun- } \\
\text { kerque, Fos, Grenoble, } \\
\text { Petite Couronne, Rouen, } \\
\text { etc.) }\end{array}$ & $\begin{array}{l}7 \text { Zones de Protection } \\
\text { Spéciale créées depuis } \\
1964 \text { (dont Paris en } \\
1964, \text { transformée et élar- } \\
\text { gie à la Petite Couronne } \\
\text { en 1978, Lyon : 1974, } \\
\text { Lille: 1974, Marseille: } \\
\text { 1981) }\end{array}$ \\
\hline $\begin{array}{l}\text { AUTRES } \\
\text { CARACTERISTI. } \\
\text { QUES }\end{array}$ & $\begin{array}{l}\text { Pour les industries im- } \\
\text { portantes, les ministères } \\
\text { de l'Industrie et de l'En- } \\
\text { vironnement intervien- } \\
\text { nent directement. La } \\
\text { procédure est définie } \\
\text { précisément par la } \\
\text { législation }\end{array}$ & $\begin{array}{l}\text { Instrument privilégié par } \\
\text { I'administration et moins } \\
\text { onéreux pour les indus- } \\
\text { tries que la Zone de Pro- } \\
\text { tection Spéciale (ZPS) }\end{array}$ & $\begin{array}{l}\text { Appliqué en priorité } \\
\text { dans les zones urbaines. } \\
\text { Pour alléger les coûts } \\
\text { importants subis par les } \\
\text { grosses unités,cet instru- } \\
\text { ment est parfois couplé } \\
\text { avec une Zone d'Alerte }\end{array}$ \\
\hline
\end{tabular}


Les acteurs principaux du système de mise en œuvre sont les 19 Directions Interdépartementales de l'Industrie (DII), devenues depuis 1981 les DRIR (Directions Régionales de I'Industrie et de la Recherche), qui sont les services extérieurs du ministère de l'Industrie. L'importance du rôle joué par ces administrations et leur indépendance vis-à-vis des niveaux central et départemental font, du système de mise en œuvre français, un système essentiellement régional et technique, si on le compare au système plutôt politique que l'on trouve dans d'autres pays européens (4). Malgré les compétences formelles des préfets, qui déliurent les actes administratifs, la mise en œuvre incombe principalement aux DII, dont les directeurs appartiennent en grande majorité au corps des mines (5). Les services des mines, que les DII ont remplacés, ont vu leurs compétences s'étendre petit à petit, le corps des ingénieurs des mines ayant su s'adapter à la diversification des interventions de l'Etat et prendre une place importante notamment pour le développement industriel, technologique et scientifique (6). Aujourd'hui, les DII sont responsables de la mise en œuvre de toute la politique régionale de développement industriel, et de tout ce qui touche à l'environnement industriel, pour lequel elles constituent comme on l'a dit parfois une «magistrature technique» (7).

Ce programme ainsi esquissé attribue à la politique régionale de mise en œuvre les cinq types de décision suivants :

- La définition de la circonscription territoriale dans laquelle va se faire l'intervention collective et son degré d'intensité.

- La combinaison des différents instruments mis à disposition, notamment le couplage entre la Zone de Protection Spéciale et la Zone d'Alerte, ou la combinaison entre les mesures prises en vertu de la ZPS ou de la ZA et les contraintes individuelles.

- Les priorités d'intervention entre les régions et a l'intérieur des régions (délais d'application des différents instruments).

- Les choix lors de l'application des instruments, notamment pour les ZPS (taux de soufre tolérés dans les fuels, période d'application de la ZPS et régime de contrôle des installations) ; pour les $Z A$, (définition des entreprises adhérentes au système d'alerte, création d'un organisme compétent pour fixer les modalités des alertes, et définition des seuils de déclenchement des alertes) ; et pour l'application des contraintes individuelles (prise en compte de l'état de l'environnement (orientation immission) ou fixa. tion des conditions d'exploitation en fonction de l'état de la technologie anti-polluante, indépendamment du taux de pollution (orientation émission). 
- La création d'un réseau de coopération entre les différents acteurs impliqués (coopération avec le niveau communal, rôle de la préfecture et importance du niveau national).

L'arrangement politico-administratif en action : le cas de trois régions choisies à tire d'exemple
Cette présentation s'appuie sur les résultats des recherches menées par une équipe de chercheurs de l'Institut d'Urbanisme de Paris, selon un cadre analytique comparatif développé par l'Institut International Environnement et Société de Berlin. Le choix des unités d'analyse de la mise en cuvre de la politique de lutte contre la pollution atmosphérique ( 3 régions et 2 à 3 localités par région) répond à une volonté de mener une analyse comparative : les unités choisies sont similaires du point de vue du taux de pollution atmosphérique (élevé), et du nombre de population exposée à cette pollution, mais différentes du point de vue des instruments utilisés, de la composition du tissu industriel et de la part relative de chaque source de pollution (8). Ont été retenues : les régions d'lle de France (Paris, Créteil, Vitry), Nord Pas-de-Calais (Dunkerque, Agglomération Lilloise), et Provence-Côte d'Azur (Fos-Etang de Berre-Martigues, Marseille). Notons enfin que cette présentation se limitera aux activités de mise en œuvre de la lutte contre la pollution atmosphérique proprement dite (étudiée durant la période 70/80) et négligera l'influence (pourtant importante) de la politique énergétique et de la conjoncture économique sur le comportement des émetteurs.

\section{La région lle de France}

Le niveau très élevé de concentration de $\mathrm{SO}_{2}$, tant dans la ville de Paris que dans la petite couronne, fait de cette région la région la plus polluée de France, même si, en terme d'évolution, on constate depuis une dizaine d'années une notable diminution de la concentration de ce polluant. Les trois zones plus précisément étudiées ont des caractéristiques différentes : à Vitry, on se trouve en présence d'un émetteur dominant, la centrale EDF, qui assure la presque totalité des émissions de $\mathrm{SO}_{2}$ de cette zone. A Créteil au contraire, l'essentiel des émissions (très faibles au demeurant) provient des chauffages urbains et domestiques. Enfin, à Paris, la situation est similaire, car il n'y a plus d'industries polluantes intra-muros, mais les émissions y sont plus importantes du fait de la forte densité des chauffages domestiques.

En 1964, on a instauré à Paris une ZPS, à l'initiative du ministère de la Santé de l'époque, afin d'organiser une première expérience de ce nouvel instrument. La mise en place d'une solution plus régionale est intervenue en 1978, lors de l'extension de la ZPS de Paris à la Petite Couronne et de l'instauration en parallèle d'une Zone d'Alerte pour les plus gros émetteurs de la Petite Couronne : l'extension de la ZPS s'est accompagnée d'un renforcement des conditions de combustion à Paris où seul le fuel TBTS ( $1 \%$ de soufre) est autorisé à l'heure 
actuelle, alors que dans la première ZPS de 1964, c'est le BTS ( $2 \%$ de soufre) qui était autorisé ; pour la Petite Couronne, l'extension de la ZPS a signifié l'obligation de consommer du fuel BTS.

Le couplage de la Zone d'Alerte et de la ZPS pour la petite couronne a été une innovation car l'utilisation conjointe des 2 instruments, n'était pas prévue par le décret de création de 1974. Ce couplage permet en fait aux établissements reliés au réseau d'alerte de ne pas respecter les prescriptions de la ZPS en été (il leur est donc possible de brûler du fuel à plus grande teneur en soufre), mais ils doivent en revanche passer au fuel TBTS lorsque l'alerte est déclenchée, c'està-dire lorsque la pollution atteint $600 \mu \mathrm{g} / \mathrm{m} 3$ d'acidité forte et/ou quand le vent est faible ; il s'agit donc de contraintes moins sévères que celles de la ZPS, qui restent cependant applicables durant I'hiver, lorsque les risques de pollution sont les plus importants. Aucune alerte n'a été déclenchée depuis que le réseau d'alerte est opérationnel (1982). Par contre, le réseau d'alerte de la centrale EDF de Vitry (qui fonctionne depuis 1973) a connu plusieurs alertes avant son rattachement au réseau général de la région.

En ce qui concerne les prescriptions plus individuelles pour les installations classées, la situation de la région Parisienne est particulière : en effet, la DII n'est pas maître de cet instrument car c'est le Service Technique des Installations Classées (STIC) de la Préfecture de Police de Paris qui en a la responsabilité (pour Paris et la Petite Couronne) ; ce service a cependant imposé aux gros émetteurs de la zone, des cheminées relativement hautes notamment pour les centrales thermiques, ainsi qu'un système individuel d'alerte pour ces dernières, à partir de 1973.

Si on essaye d'évaluer l'impact des mesures prises, il faut nuancer en fonction des zones étudiées : pour Paris, il est certain que la ZPS de 1964 a nettement réduit les émissions et les immissions de la zone ; la transformation de cette première ZPS en 1978 semble elle aussi avoir été efficace, car la qualité de l'air s'est encore améliorée ces dernières années, pour atteindre $71 \mu \mathrm{g} / \mathrm{m} 3$ en 1981. Ce taux de pollution relativement élevé peut cependant être considéré comme une réussite, vu la forte pollution de la zone au début de la période d'observation $(120 \mu \mathrm{g} / \mathrm{m} 3)$. A Vitry comme on pouvait s'y attendre, l'introduction du système d'alerte pour le principal émetteur dès 1973 n'a pas fait baisser les émis. sions de soufre, qui ont tendance à augmenter durant la période considérée. Par contre, ce système d'alerte qui a "bien» fonctionné durant cette période (22 alertes en 1980) a permis d'écrêter les pointes de pollution et aussi d'assurer une meilleure qualité de l'air, en terme de pollution moyenne ; cette amélioration de la situation est également due à la construction de hautes cheminées pour la centrale thermique EdF, dès 1972, qui permettent une bonne dispersion des polluants, et à des conditions climatiques moins défavorables ces dernières années. A Créteil enfin, on ne peut pas imputer l'amélioration générale de la 
qualité de l'air de cette zone à l'introduction de la ZPS en 1978, car les émissions comme les immissions ont baissé pour cette zone avant même l'introduction de la ZPS. II semble que la crise économique (ralentissement économique) et que les économies d'énergie réalisées dans cette zone soient largement plus responsables de l'amélioration constatée.

En conclusion, on peut dire que la politique menée dans cette région est globalement positive, notamment pour les zones les plus polluées où la qualité de I'air s'est nettement améliorée. Mais la ZPS semble avoir mieux fonctionné que le système d'alerte, car les niveaux de pollution des zones où elle a été introduite ont plus baissé que dans les zones où une ZA a été introduite.

\section{La région Nord Pas-de-Calais}

Cette région est fortement émettrice de $\mathrm{SO}_{2}$ du fait de l'implantation d'industries particulièrement polluantes, auxquelles s'ajoute la présence de centrales thermiques EdF. Par contre, en moyenne, les immissions de cette région ne sont pas particulièrement élevées. Les deux zones que nous avons plus précisément étudiées à l'intérieur de cette région sont assez contrastées; on trouve une zone fortement émettrice, la zone de Dunkerque, mais peu polluée en terme d'immission, tandis que l'agglomération lilloise est nettement moins émettrice mais avec des taux d'immissions bien plus élevés. Cela s'explique par une structure différente des émetteurs : il y a prédominance de gros émetteurs à Dunkerque (centrale thermique, raffinerie, sidérurgie), bien qu'en faible nombre, qui entraîne des émissions importantes, mais des immissions beaucoup plus faibles du fait d'une bonne localisation des émetteurs (littoral) et de cheminées très hautes ; en revanche, on enregistre des pointes de pollution très importantes dans cette zone. A Lille par contre, on est en présence de beaucoup de petits émetteurs (petites et moyennes industries, chauffage urbain et surtout chauffages domestiques), ce qui explique les faibles émissions, mais qui, disséminés dans toute la ville et jusqu'en 1974, sans aucun contrôle ou presque, provoquaient un taux de pollution important.

La politique menée par la DII en matière de contrôle des installations industriel. les est ancienne; cette Dil a été une des premières à prendre en charge l'application de la législation des installations classées et en matière de pollution atmos. phérique, elle a donc pu jouer sur les trois instruments pour mettre en place une politique globale de lutte contre la pollution atmosphérique.

La contrainte individuelle est plus marquée à Dunkerque, du fait de l'existence de gros émetteurs ; cela répond à une volonté du gouvernement national de contrôler prioritairement ces grosses unités. Mais ce contrôle individuel est plus informel qu'à Lille du fait d'une bonne relation entre les ingénieurs des mines et les responsables industriels. Ce qui a permis l'introduction progressive à 
Dunkerque de hautes cheminées assurant une meilleure dispersion des polluants dans l'atmosphère. Mais le tissu industriel de Dunkerque étant relativement ancien et connaissant des difficultès financières (la sidérurgie notamment), la DII n'a pas pu imposer aux installations des investissements très importants pour lutter contre la pollution de l'air.

A Lille, le contrôle des établissements porte plus (depuis l'introduction de la ZPS en 1974) sur les combustibles utilisés et les conditions de combustion des installations, les prescriptions des arrêtés préfectoraux étant par ailleurs appliquées (hauteur des cheminées). En ce qui concerne l'utilisation des deux autres instruments, nous nous trouvons devant deux politiques différentes :

- A Lille, l'introduction de la Zone de Protection Spéciale, en 1974, s'est faite à la suite de l'expérience parisienne et en même temps que la ZPS de Lyon. Le fuel autorisé est le fuel BTS ( $2 \%$ de soufre). En 1981, on a mis en place également une Zone de Surveillance et d'Alerte; lors de cette création, on a parlé d'une possibilité de couplage entre la ZPS et la $Z A$, qui permettrait à certaines installations de déroger aux prescriptions de la ZPS, mais il semble que cette possibilité ait été abandonnée à l'heure actuelle.

- A Dunkerque, on a introduit, en 1977, une Zone d'Alerte qui a commencé à fonctionner réellement en 1981 ; les alertes déclenchées en fonction des prévisions météorologiques varient selon les années (22 en 1981 et 7 en 1983).

En terme d'impact de la politique mise en place par la DII, nous pouvons parler d'une incidence positive de la ZPS de Lille ; les émissions ont fortement diminué à partir de 1977 et la qualité de l'air s'est nettement améliorée, pour atteindre même $54 \mu \mathrm{g} / \mathrm{m} 3$ en 1981. Par contre à Dunkerque les émissions de $\mathrm{SO}_{2}$ ont légèrement diminué durant la période considérée, mais l'introduction de la Zone d'Alerte ne semble pas en cause, car la baisse des émissions est intervenue avant son entrée en vigueur. Ici aussi, le ralentissement économique, ainsi que la conversion de certaines industries au gaz naturel moins polluant, semblent à l'origine de cette baisse. La qualité de l'air ambiant à Dunkerque est assez satisfaisante, mais on peut remarquer durant la période considérée une légère augmentation de la pollution moyenne lavec une pointe à $46 \mu \mathrm{g}$ en 1979), bien que la situation semble s'être stabilisée durant ces 2 dernières années ; par contre les pointes de pollution ont été évitées dès la mise en place du réseau, bien que cela ne se fasse pas sentir en terme de pollution moyenne. 


\section{La région Provence Côte-d'Azur (9)}

Cette région est la région la plus émettrice de $\mathrm{SO}_{2}$ de France; comme dans le Nord, cette situation s'explique par la présence d'industries fortement émettrices. La situation des deux zones que nous avons particulièrement étudiées à l'intérieur de cette région est elle aussi contrastée : on trouve une zone industrielle (Fos), fortement émettrice mais peu polluée et une zone urbaine (Marseille), plus faiblement émettrice mais avec une qualité de l'air plus dégradée. Ici aussi, la présence d'émetteurs structurés différemment Igros émetteurs a Fos et dissémination de petits émetteurs à Marseille) explique ces différences de situation.

La politique suivie dans ces deux zones s'apparente à celle que nous venons de voir pour le Nord : on a créé à Fos, en 1978, une Zone d'Alerte, opérationnelle depuis 1979, et une ZPS a Marseille en 1981 ; cette ZPS n'autorise la consommation que du fuel TBTS ( $1 \%$ de soufre), mais en revanche cette prescription n'est obligatoire que durant I'hiver. On a également introduit une mini-zone d'alerte autour de la centrale thermique de Gardanne, qui devra en cas d'alerte réduire son activité. Du point de vue de la mise en place d'une politique globale de lutte contre la pollution, la situation de Fos est particulière : la création de la zone industrielle de Fos est un projet national, et une structure particulière a été créée en 1971 : le Secrétariat Permanent Pour les Problèmes de Pollution Industrielle (SPPPI) qui a été chargé de mettre en place une politique globale de lutte contre la pollution notamment atmosphérique. Cette structure prolonge en fait l'action de la DII, tout en tenant compte des contraintes nationales ici imposées.

Du point de vue des prescriptions individuelles, la législation des installations classées est appliquée de façon plus contraignante qu'à Dunkerque ; la DII a mis en place depuis 1973 une politique de réduction des émissions de soufre dans l'atmosphère, sur l'ensemble de la zone industrielle. Dans ce but, la DII a obligé les installations émettrices de $\mathrm{SO}_{2}$, dans le cadre de la législation des installations classées, à s'équiper d'installations de récupération du soufre des gaz effluents. La DII par la biais du SPPPI a défini dès 1973 un seuil d'émission de 800 tonnes de $\mathrm{SO}_{2}$ par jour à ne pas dépasser sur l'ensemble de la zone. Ce quota global a été ensuite réparti entre les industries, qui ont dû, pour res. pecter leur quota individuel, installer petit à petit des équipements de récupération de soufre (12 industries en sont équipées à l'heure actuelle). Ces mesures ont été efficaces, car de 750 tonnes de $\mathrm{SO}_{2}$ émis par jour en 1973, on est arrivé aujourd'hui à 350 tonnes par jour. Parallèlement à ces mesures, la DIl a mis en place une Zone d'Alerte, pour éviter les pointes de pollution parfois très importantes en hiver (déclenchement des alertes en fonction des conditions météorologiques). Cependant, ce réseau d'alerte doit être de nouveau adapté pour tenir compte de la baisse des émissions de la zone. 
A Marseille, dans le cadre de la législation des installations classées, la Préfecture joue un rôle plus important car politique face à une mairie dont le poids n'est pas négligeable; la Préfecture est une sorte de relais entre les industries, les collectivités locales et la DII, pour les questions délicates, mais l'expertise technique reste du ressort de la seule DII.

En terme d'impact de ces politiques, on peut constater que, pour l'ensemble de la région comme pour les deux zones étudiées, la situation s'est améliorée durant ces dernières années; dans le cas de Fos, depuis que le réseau d'alerte fonctionne, il n'y a eu que deux alertes liées à des conditions climatiques défavorables. La politique de réduction des émissions de soufre a été suffisamment efficace pour transformer le réseau d'alerte en un réseau de surveillance principalement. A Marseille l'introduction de la ZPS a eu un impact plus positif, puisque la pollution moyenne a nettement diminué à partir de 1981 pour atteindre $56 \mu \mathrm{g} / \mathrm{m} 3$ en 1983. Mais il est vrai que les économies d'énergie ont joué elles aussi un rôle, car tant les émissions que les immissions ont baissé avant même l'introduction de la ZPS. D'autre part, l'introduction de cette ZPS est intervenue très tard si I'on compare avec la situation des autres villes en France (à degré de pollution et de poputation comparable), ce qui permet de modérer la réussite que nous avons constatée ; d'autant plus que la DII est intervenue dans le cas de Fos dès 1973, alors que la situation de cette zone (taux de pollution et de population) ne le justifiait pas expressément. On constate donc une disproportion entre les moyens employés et la pression du problème dans ces deux zones.

\section{Evaluation comparative des trois politiques régionales}

On peut conclure face aux situations régionales que nous venons de présenter que, dans l'ensemble, l'introduction des ZPS a été dans tous les cas étudiés particulièrement efficace pour l'amélioration de la qualité de l'air de ces villes, et que par contre, le système d'alerte n'a pas eu une efficacité aussi radicale. Le couplage entre ZA et ZPS ne peut pas, quant à lui, être réellement évalué car il est trop récent. Toutefois la première expérience dans la Petite Couronne montre une certaine efficacité pour la réduction de la pollution de l'air. II sem. ble cependant que si on introduit un couplage pour les ZPS déjà créées, cela risque d'amoindrir l'efficacité constatée de ces ZPS.

Par ailleurs, il ressort de la description que nous venons de faire, que malgré l'utilisation d'instruments d'intervention semblables, les politiques sont différentes d'une région à l'autre (modalités d'application et impacts différents) : pour les quatre ZPS, on constate des différences d'adaptation de cet instrument aux spécificités régionales : la ZPS de Lille comme celle de la Petite Couronne comportent une restriction moins sévère que celle de Paris du point de vue des fuels autorisés, et la ZPS de Marseille est modulée en fonction de la période de l'année. 
Il faut remarquer également les différences dans les délais d'intervention : très rapide pour la région parisienne, l'introduction de la ZPS a été particulièrement tardive à Marseille notamment en comparaison avec Lille.

Pour le couplage ZPS/ZA, dans le cas où il est envisagé ou réalisé, il n'a pas la même signification : instrument dérogatoire dans le cas de la Petite Couronne, il apparaît comme complémentaire dans le cas de Marseille (pour la centrale de Gardannel. Dans le cas de Lille, l'idée de couplage semble abandonnée, mais si elle était maintenue elle pourrait être une mesure plus sévère (ZPS avec fuel BTS à $2 \%$ et fuel TBTS à $1 \%$ durant les alertes) ou une mesure dérogatoire comme dans le cas de la Petite Couronne.

Si on compare les deux Zones d'Alerte, on constate ici aussi des différences notamment sur le seuil de déclenchement des alertes; seuil de pollution précis dans la Petite Couronne, il dépend beaucoup plus des conditions météo à Dunkerque, et des conditions locales de pollution à Fos. Le nombre d'alertes étant d'ailleurs totalement variable d'une zone à l'autre.

En terme d'impact, si on se fonde sur les seuils définis par la CEE, on peut constater également des différences entre ces trois régions. Parmi les zones urbaines dotées d'une ZPS que nous avons étudiées, seule Lille est au dessous de la norme, la région parisienne et Marseille dépassant les seuils de pollution de pointe et de fond; sachant par ailleurs que les niveaux de pollution de ces zones étaient equivalents en 1970 (autour de $120 \mu \mathrm{g} / \mathrm{m} 3$ ), on peut conclure que l'introduction des ZPS a eu un impact différent dans les trois régions, plus efficace à Lille que dans les deux autres zones ; cette conclusion est cependant à tempérer par le fait que l'émission de $\mathrm{SO}_{2}$ est moins importante sur l'ensemble de la période à Lille qu'à Marseille ou Paris. On peut faire la même remarque pour les zones d'alerte ; si Fos apparaît nettement plus au dessus des normes CEE que Dunkerque, il faut souligner que l'émission $v$ est 5 fois plus importante. On peut donc retenir que dans les deux zones, la situation a été améliorée, mais que dans la zone de fos cette amélioration a été plus sensible (la pollution moyenne y a nettement diminué, contrairement à Dunkerque). Les délais d'intervention ont par contre été similaires.

Les facteurs explicatifs des différences observées entre les politiques régionales

\section{Le modèle explicatif}

11 existe un large consensus, parmi les politistes qui ont mené des recherches sur la mise en œuvre de politiques publiques, sur la définition des facteurs susceptibles d'affecter la qualité de la mise en cuvre d'une politique publique : on distingue normalement, la structure du programme (normalement national), le poids économique, social et politique des administrés («implementation field»), et le système administratif de mise en œuvre , y compris le réseau 
d'intéraction («implementation structure»). Dans la présentation qui suit, nous tenons compte de ce schéma général, mais nous ne reprendrons pas la dimension "structure du programme" car, dans le cadre de la politique de lutte contre la pollution de l'air, les éléments substantiels sont élaborés au niveau de la politique de mise en œuvre elle-même. Lors de l'analyse comparative des recherches menées dans les 5 pays inclus dans la comparaison internationale (RFA, France, Italie, Royaume Uni, Pays Bas) les indicateurs suivants se sont avérés les plus pertinents pour caractériser l'apport explicatif des deux dimensions restantes («implementation field» et «implementation structure»)(10) :

- La pression objective du problème mesurée par les taux d'immission et leurs évolutions dans le temps ainsi que le nombre d'habitants exposés à cette pollution. Cela est d'autant plus vrai pour la politique française que celle-ci (au moins jusqu'au début des années 80 ) a été orientée vers le contrôle des immissions. Cet indicateur de base apparaît explicatif de l'intervention elle-même, de son intensité et du moment choisi pour intervenir.

- La structure des sources de pollution explique par contre le choix des instruments d'intervention et l'intensité de cette intervention. Nous distinguerons entre les zones industrielles (nombre limité de sources de po!lution, mais émissions importantes), et les zones urbaines (nombreuses sources d'émission, domestiques et artisanales). A l'intérieur des zones industrielles nous pouvons distinguer entre un tissu industriel ancien et un tissu composé d'entreprises relativement modernes. La structure des sources de pollution est le premier facteur susceptible d'accélérer ou de freiner l'intervention publique telle que demandée par la pression du problème. Cette explication est valable pour les différences entre les régions comme pour les différences entre les localités d'une même région.

- La constellation des acteurs et leurs stratégies seront considérées comme un indicateur (relativement complexe) de la capacité explicative de la structure de mise en œurre, qui affecte les caractéristiques de l'intervention publique dans les différents espaces examinés de plus près (intensité, moment d'intervention, choix des instruments et modalités de leur application). Ces constellations d'acteurs qui entourent les DII, ainsi que leurs stratégies, peuvent elles aussi accélérer ou freiner l'intervention publique de la DII. Elles peuvent attirer l'attention des pouvoirs publics sur les problèmes de pollution atmosphérique de certaines zones, ou au contraire, leur donner une image trop positive de la situation d'autres zones. L'influence des acteurs est présente également lorsqu'il s'agit de formuler les mesures concrètes à prendre, et d'évaluer l'adéquation et l'impact de ces mesures à la situation locale. 
- L'indépendance des DII vis-à-vis des acteurs impliqués à l'intérieur du réseau d'intéraction est le dernier facteur déterminant. Une telle indépendance est liée à l'existence d'une capacité analytique importante (en terme de personnel, d'instruments et de budget), et d'une capacité de négociation. Plus l'indépendance des DII, garantie par un personnel qualifié et compétent, sera grande, moins l'intervention publique sera gérée selon des critères politiques ou partiaux (qui peuvent favoriser certains espaces au détriment d'autres). Qaund de tels critères entrent en compte, cela mène à l'attribution de positions privilégiées pour ceux qui crient le plus fort, sans rapport avec la situation réelle de l'environnement.

L'analyse qui suit présente les traits principaux de ce modèle explicatif appliqué aux trois régions particulièrement étudiées.

\section{La région lle de France}

Cette région connaît un problème de pollution et une population particulièrement importants qui apparaissent nettement explicatifs du choix de la DII d'imposer une ZPS pour l'ensemble de la zone. L'intervention de l'administration dès 1964 apparaît justifiée par les facteurs pollution et population. Cependant le choix des moyens d'intervention s'explique par d'autres facteurs : l'introduction de la ZPS à Paris est une réponse adaptée à la structure des sources de pollution (nombreux petits émetteurs échappant aux contraintes individuelles). Par contre, l'extension de la ZPS à la Petite Couronne n'est pas une réponse aussi adéquate, car le tissu urbain et industriel est extrêmement mêlé. La résistance des gros émetteurs de cette zone à l'introduction de la ZPS explique le retard de cette extension (qui n'est intervenue qu'en 1978, soit 4 ans après les ZPS de Lille et de Lyon, alors que le degré de pollution de la Petite Couronne était aussi important). Les gros émetteurs touchés par les contraintes individuelles, plus adaptées à leurs cas, refusaient la mesure collective. Le couplage de la ZPS avec la ZA a été plus influencé par les stratégies développées par les ministères de l'Industrie et de l'Environnement. En effet, c'est à cause de l'intervention du Ministère de l'Industrie que les centrales EDF situées autour de la région parisienne ont dû installer un réseau de mesure devenu à partir de 1973 un réseau d'alerte. Aussi, lorsqu'il a été question d'étendre la ZPS de Paris à la Petite Couronne, les centrales EDF ont demandé à déroger aux prescriptions de la ZPS, du fait du système d'alerte auquel elles se soumettaient. Le Ministère de l'Environnement et celui de l'Industrie ont été au centre de la négociation pour le couplage ZPS/ZA là remarquer le poids déterminant du corps des mines, qui se trouve au niveau central, au sein des deux ministères). Mais les responsables des industries de la région, regroupés en une association de défense de leurs intérêts, sont également intervenus dans le 
processus, pour négocier leur participation au réseau d'alerte et les possibilités de déroger à la ZPS ; deux types de négociation sont intervenus :

- d'une part, une négociation entre les deux ministères (Industrie et Environnement) et le CITEPA, un organisme technique représentant les intérêts des industries en général en matière de pollution de l'air ; cette négociation a porté sur le principe du couplage ZPS et ZA, accepté finalement par le Ministère de l'Environnement.

- d'autre part entre la DII et l'association régionale des industriels pour les problèmes de pollution atmosphérique (AIRASIF), pour la détermination des conditions pratiques de ce couplage, et notamment la détermination du seuil de déclenchement des alertes.

Le cas de la région parisienne illustre parfaitement la forte influence que peut avoir le niveau central sur une administration régionale. II faut remarquer, par ailleurs, l'absence de participation active des collectivités locales de cette région, principalement due à des dissensions politiques entre les mairies de Paris et de la Petite Couronne et à la participation d'acteurs dominants comme les ministères qui remplace en partie celle des autres acteurs. Cette participation très importante du niveau central ne doit pas faire oublier le rôle non négligeable de la DIl lors de la négociation du seuil de déclenchement des alertes ; la politique de la DII repose finalement sur la négociation de ce seuil avec les industriels, car c'est de lui que dépend le nombre d'alertes et donc les coûts de la lutte contre la pollution de l'air à supporter par les industries. Le seuil retenu de $600 \mu \mathrm{g}$ correspond au seuil que les centrales thermiques EDF avaient déterminé pour leur propre réseau, avant leur intégration dans le réseau général de la région parisienne ; c'est un seuil relativement sévère, comparé à d'autres pays (11), qui s'explique par la situation de la pollution et de l'urbanisation de cette région. La création d'une association de gestion du réseau d'alerte, proposée par la DII (AIRPARIF) a permis de faire financer le réseau d'alerte par les industries et les collectivités locales (elles ont finalement accepté d'en faire partie). La création de cette association, inspirée de l'expérience du SPPPI de Fos, a surtout permis à la DII, de rassembler autour de son projet de réseau, tous les acteurs potentiels pouvant intervenir, afin de gérer l'ensemble du problème régional de pollution de l'air.

Au vu des résultats de cette politique, on peut admettre que les solutions choisies ont été plus influencées par la structure même du problème de la pollution que par les stratégies des acteurs. Plus précisément on peut remarquer que la concentration de l'action administrative sur la ville de Paris, dans les années 60 , s'explique essentiellement par le fait qu'à l'époque les taux d'immission de cette ville étaient beaucoup plus élevés que dans la Petite Couronne; par contre l'extension tardive de la ZPS, dont la nécessité se manifestait dès le début des années 
70, montre l'influence importante des acteurs économiques de la zone, qui ont freiné la politique de la DII, et l'absence des acteurs locaux qui auraient pu faire contrepoids. A partir des années 80 , on assiste à une politique plus équilibrée du fait de la position renforcée de la DII.

\section{La région du Nord Pas-de-Calais}

Dans les zones étudiées de cette région, la pression du problème est nettement moins importante que précédemment, car la qualité de l'air y est moins dégradée ; cependant, le degré de pollution a eu une influence certaine sur le moment d'intervention et sur le choix des instruments retenus. Cela se manifeste notamment par le caractère différent de l'intervention de la DII :

A Lille, le degré de pollution a en effet influencé la création de la ZPS ; cette création faisait partie du programme national de lutte contre la pollution de l'air, qui tente de privilégier les zones urbaines peuplées et polluées. Pour Lille, comme pour Lyon, on trouve donc exceptionnellement un impact relativement direct du programme national, édicté au cours de la même année (1974). Cependant l'introduction de cette ZPS est également due à une constellation favorable d'acteurs et à leurs stratégies, qui ont accéléré l'intervention collective : on se trouve, en effet, en présence de collectivités locales conscientes du problème de pollution atmosphérique, notamment sous la pression de I'APPA lune association de médecins et d'hygiénistes ayant pour but de développer les réseaux de mesure de la pollution de l'air en France) qui tente de sensibiliser l'opinion sur les problèmes de pollution de l'air ; le Conseil Général comme les municipalités et la Communauté Urbaine de Lille ont soutenu financièrement le développement d'un réseau de mesure dès les années 1970. Cette intervention des collectivités locales explique aussi que cette ville ait été dotée d'une ZPS dès 1974 (alors que Marseille a attendu jusqu'en 1981), et que la DII ait imposé des prescriptions relativement sévères aux industries dans le cadre de la législation des installations classées (notamment des hautes cheminées). La résistance des industriels de la région a l'introduction de la ZPS a été assez faible; ils ne sont pas regroupés au sein d'une association défendant leurs intérêts en matière de pollution atmosphérique comme c'est le cas à Dunkerque par exemple. De plus, la taille de ces industries, relativement petite, ne leur confère pas individuellement un poids économique suffisant pour négocier avec une DII qui, elle, a une capacité technique et de négociation importante. Une enquête récente montre d'ailleurs que peu d'entreprises ne respectent pas les prescriptions. Comme en région parisienne, les industriels se sont vus proposer par la DII, de participer à une association de gestion du réseau de mesure, qui ras- 
semble à la fois les industriels, les collectivités locales et même certaines organisations de protection de l'environnement, et qui permet à la DII de gérer le problème de la pollution de l'air.

A Dunkerque, le degré de la pollution moyenne, relativement bas, ne demandait pas l'introduction d'une ZPS ; la structure des émetteurs ainsi que la pollution de pointe parfois très forte, requéraient par contre, dès le début des années 70 , l'introduction d'une zone d'alerte et des contrain. tes individuelles importantes. Mais la situation économique des industries rend fort difficile toute demande d'investissements supplémentaires. Quant à la ZA, les industriels ont exercé une pression très forte sur la DII. Leur importance pour l'économie régionale et nationale leur a conféré un poids de négociation non négligeable. De plus, ils se sont regroupés en une association de défense de leurs intérêts en matière de pollution de l'air dès 1977, lorsque la DII a émis l'idée de création d'une ZPS. Dans ces conditions, la DII a dû manœuvrer avec souplesse, sa capacité à imposer certaines prescriptions étant plus faible qu'à Lille par exemple. Cette pression des industriels fait que le jeu s'est joué à deux partenaires essentiellement : la DIl et les industriels, les autres acteurs n'intervenant que très faiblement. Ni les collectivités locales, dont le développement économique dépend assez fortement de la présence de ces industries, ni l'APPA ou d'autres organisations de défense de l'environnement, dont les mem. bres (la population) dépendent eux aussi de ces industries, n'ont su (ni voulu) s'interposer entre ces deux partenaires. La DII a fait rentrer dans le jeu les autres acteurs concernés (les collectivités locales et les associations de défense de l'environnement) dans le cadre d'une association de gestion du réseau de mesure et d'alerte, qui lui permet de gérer l'ensem. ble du problème, et aussi d'imposer une solution plus technique (un réseau d'alerte entièrement informatisé).

En conclusion, on peut retenir que la capacité explicative de la pression et de la structure du problème, pour l'action de la DII, est faible dans les deux zones, car des constellations d'acteurs favorables (Lille) ou opposées (Dunkerque) ont influencé l'intervention administrative. La DII, bien que l'une des plus importantes de France, a eu du mal à se détacher de ces pressions politiques opposées.

\section{La région Provence Cóte-d'Azur}

Nous pouvons constater, ici aussi, une nette différence de l'intervention publique entre les deux zones analysées. Mais dans ce cas, la différence joue dans le sens inverse. Le poids fort de l'action de la DII durant les années 70 s'est concentré sur Fos, pourtant moins pollué et moins peuplé que Marseille, qui elle échappe à un contrôle effectif jusqu'en 1981. Mais ici, la pression du problème apparaît 
moins explicative de la distribution spatiale de l'intervention publique, que la structure des sources de pollution (tissu industriel relativement moderne et donc à même d'installer des équipements anti-pollution, et tissu ancien à Marseille, composé d'installations de chauffage domestique, artisanales et industrielles) ; il faut noter, d'autre part, une variation considérable de la constellation des acteurs qui ont permis, dans le cas de Fos, d'accélérer une intervention administrative (que l'on peut même qualifier d'excessive, en comparaison avec la situation du pays) et qui par contre, dans le cas de Marseille, ont freiné une action administrative que l'on peut considérer comme une des plus faibles de l'hexagone durant les années 1970. Plus précisément, la situation de chacune des 2 zones se caractérise ainsi :

A Fos, l'influence du gouvernement central s'est fait particulièrement sentir ; en effet, le projet de la zone industrielle de Fos, étant un projet national, la DIl comme le Préfet, n'ont joué qu'un rôle administratif lors des premières demandes d'autorisation, le ministère de l'Industrie, assurant à leur place l'expertise et la prévision des installations du point de vue technique. Mais avec la création du SPPPI, la pollution est devenue non plus un problème individuel, mais un problème global, nécessitant une solution globale. Le SPPPI a été créé à la suite du rapport Schnell, lui-même sollicité par le Ministère de l'Industrie, car le maire de Fos, qui s'était vu refuser la possibilité d'étendre la construction sur sa commune sous prétexte de pollution, a posé au gouvernement la question de la pollution de l'ensemble de la zone industrielle. En réponse à cette pression, le gouvernement a dépêché sur place une mission interministérielle chargée "d'étudier la situation» et de proposer des solutions. Le rapport de la mission Schnell a préconisé la création du SPPPI, chargé de proposer et de mettre en œuvre une politique globale dans le domaine de l'eau et de l'air. La pression des collectivités locales et du gouvernement national a permis à la DII d'intervenir de façon particulièrement contraignante en imposant un système de réduction des émissions. Cette mesure, unique en France, a été rendue possible, par le fait que l'on a "construit» volontairement la zone industrielle de Fos, en tant que projet d'envergure nationale. La DII a pu également imposer une ZA permettant de limiter les pointes de pollution en attendant que les mesures de limitation des émissions soient opérationnelles. Mais la DII a également appuyé sa politique sur une structure de concertation particulière, le SPPPI.

- A Marseille, l'introduction de la ZPS a reçu une forte opposition de la part des industriels, ce qui a considérablement retardé le processus de décision. La DIl et le préfet ont dû négocier l'application de la ZPS avec les industries qui sont encore nombreuses à l'intérieur de la ville. De cette négociation est sorti un aménagement de la ZPS, qui ne sera finalement applicable qu'en hiver. La résistance des industriels de l'agglomé- 
ration de Marseille s'explique en partie par le fait qu'ils se sont regroupés en une association chargée de défendre leurs intérêts en matière d'environnement. Le rôle du préfet a été en ce sens important, car il a servi d'arbitre entre la DII et les industriels dans la négociation autour de la mise en place de la ZPS (dans laquelle est également intervenu le CITEPA à la demande de l'association des industriels). Le préfet est intervenu aussi pour négocier et accorder des délais de réalisation lors de la procédure d'autorisation d'installations classées. Ce rôle du préfet est dans le cas de Marseille une conséquence de la présence d'une mairie dont le poids politique et la capacité de négociation (soutenue par un service municipal développé) sont importants, notamment dans le cadre du Conseil Départemental d'Hygiène. II s'agit d'une complémentarité entre la DII et la préfecture, que nous n'avons pas trouvée dans les autres cas étudiés.

\section{Comparaison des modèles explicatifs}

Dans le cadre de l'arrangement politico-administratif de la lutte contre la pollution de l'air en France, les trois régions ont développé des politiques de mise en cuvre, sensiblement différentes du point de vue de la distribution de l'interven. tion publique dans l'espace. Nous avons essayé d'expliquer ces modèles de distribution par différents facteurs : la distribution spatiale de la pression du problème, la variation de la structure des émetteurs de la pollution, la variation de la constellation et des stratégies des acteurs dans les différents espaces, et les caractéristiques des trois DII. II s'avère que parmi les trois modèles explicatifs, dans un cas (région lle de France), la pression du problème et la structure des sources dominent nettement les facteurs liés à une variation de la constellation des acteurs. Les conditions trouvées dans ce cas permettent donc le développement d'une politique de mise en œuvre avec peu de "déviances», par rapport à la distribution spatiale de la pression du problème (évolution des taux d'immission, importance de la population exposée dans Paris et la Petite Couronne). L'efficacité de cette politique de mise en œuvre se caractérise par une action relativement intensive, sur tous les espaces étudiés, par le respect de l'objectif de la lutte contre la pollution atmosphérique d'une manière satisfaisante sur l'ensemble de cette région, et par une certaine "technicité».

Les conditions de fonctionnement de cette politique dépendent essentiellement de plusieurs facteurs :

- la position très particulière de la région parisienne, quelle que soit la politique publique (condition indépendante de la politique de l'environnement) ; 
- la constellation équilibrée des acteurs (poids relatifs, portée régionale et non pas locale) :

- le fait qu'aucun des acteurs ne voulait ni ne pouvait à lui seul s'opposer aux actions envisagées ;

- une large concertation entre les deux ministères nationaux impliqués (Environnement et Industrie) :

- l'absence d'acteurs locaux qui auraient, soit freiné, soit fait accélérer l'intervention dans des espaces particuliers ;

- l'absence de toute politisation de la gestion de l'environnement et surtout - vers la fin des années 70 . l'indépendance de la DII qui lui a permis de contrôler le réseau des acteurs au sein de l'association de gestion du réseau de mesure.

L'intervention des pouvoirs publics dans le Nord et dans les Bouches du Rhóne se distingue de celle de la région parisienne par une intervention différenciée selon les zones étudiées. Pour les quatre zones analysées, cette distribution inégalitaire de l'intervention publique ne s'explique que partiellement par la structure différente des sources de la pollution (zones industrielles à Dunkerque et Fos, zones urbaines à Lille et Marseille), et ne s'explique pas non plus par une variation de la pression du problème (taux de pollution et concentration démographique). L'explication principale de cette distribution inégalitaire réside par contre dans l'existence de constellations d'acteurs différentes dans ces zones et dans les processus politiques d'intéraction entre ces acteurs et les DII. Dans les deux régions, les acteurs locaux ont joué un rôle beaucoup plus important que dans le cas de la région parisienne. Ces acteurs locaux ont été capables de former une coalition avec le niveau central (cas de Fos et - cas inverse - de Dunkerque) pour attirer une intervention publique précise, ou pour se protéger de cette intervention. La DII dans les deux cas a eu de la peine à conserver son indépendance, vu le fort degré de politisation (cas des Bouches du Rhône) ou tout au moins moyen (cas du Nord).

C'est ici que la ressemblance entre les deux modèles explicatifs s'arrête. Comme nous l'avons vu, la constellation des acteurs et leurs stratégies dans le cas $d u$ Nord n'ont pas ernıêché une distribution adéquate de l'intervention publique par rapport à la pression du problème à l'intérieur du département du Nord. Les activités de la Communauté Urbaine lilloise ont au contraire accéléré l'intervention là où elle était nécessaire, sans pour cela freiner l'intervention dans la zone de Dunkerque qui connaissait une pression de problème moins forte à l'époque. Cette constellation des acteurs a accéléré, dans le Nord, une politique de mise en œuvre adaptée à la poursuite des objectifs nationaux de lutte contre 
la pollution de l'air. Par contre dans les Bouches du Rhône, une constellation d'acteurs, a mené à une distribution de l'intervention publique très inadéquate vis-à-vis de la pression du problème. La forte concentration des activités de la DIl sur le pôle industriel de Fos, déclenchée par les pouvoirs locaux qui ont réussi à former rapidement une coalition avec le niveau national, n'est pas justifiée par la pression du problème malgré tout modeste (immissions et population exposée plus faibles), comparée à l'absence d'une intervention efficace à Marseille, troisième ville de France et fortement polluée. II est intéressant de noter que l'intervention à Fos, dès les années 70, a été motivée par la DII, par une nécessité de réorienter la politique de lutte contre la pollution de l'air, vers le contrôle des émissions et non plus vers le contrôle des immissions. L'orientation du programme national doit donc être changée au moins officieusement pour justifier (ex post ?) l'intervention publique dans ce pôle industriel de portée nationale.

Conclusions : l'apport de l'analyse à la politique de l'environnement en France et à la théorie des politiques publiques (12)
L'analyse de la politique contre la pollution de l'air, telle que présentée, nous permet de tirer quelques conclusions quant à la conception future de cette politique. Nous développerons ensuite des aspects plus généraux qui contribuent à la théorie de l'analyse de politiques publiques, car ils devraient se retrouver dans d'autres politiques publiques également. Ils porteront sur l'effet distributif des politiques publiques régulatrices et l'importance du facteur politique local pour la conception de la mise en œuvre de politiques publiques.

- Conséquences pour la conception d'une politique future de lutte contre la pollution de l'air : la question qui se pose est comment éliminer l'écart, constaté dans l'analyse présentée, entre la distribution spatiale de la pression du problème et celle de l'intervention publique. II faut préalablement remarquer que d'une part, parmi les 14 régions européennes analysées (13), les trois régions françaises se situent parmi les régions dont la politique de mise en œuvre est des plus équilibrées par rapport au problème de pollution ; la question ne se pose en fait, en France, que pour le cas des Bouches du Rhône durant les années 70. D'autre part, la correspondance entre la distribution spatiale du problème et celle de l'intervention publique ne peut être avancée que pour le moyen terme, car il est souvent raisonnable de concentrer l'intervention publique dans les espaces où elle ne se heurte pas à des difficultés insurmontables, même si d'autres espaces nécessitent une intervention plus rapide. En tenant compte de ces deux réserves, on peut avancer deux stratégies différentes pour obtenir une intervention publique plus équilibrée. La première, sollicitée surtout par les politiciens, consisterait à renforcer les éléments contraignants du programme national. Un ren. forcement des éléments substantiels de ce programme pourrait améliorer l'application des contraintes individuelles (standards techniques minimaux pour toutes 
les installations de combustion, applicables sur l'ensemble du territoire, indépendamment de la qualité de l'air ambiant ; réduction du taux général de soufre dans les fuels lourds et domestique). De telles mesures s'imposeront dans un futur proche, car la seule politique susceptible de s'attaquer aux causes de la pollution atmosphérique et - surtout - de combattre le dépérissement des forêts et de la faune situées à une grande distance des sources de pollution (14). est une politique réorientée vers un contrôle systématique des émissions. Par contre, un renforcement des éléments du programme national relatifs à la politique de mise en œuvre des DII dans les zones polluées, doit être considéré avec scepticis$m e$. Les recherches menées en RFA et en Italie (15) nous ont amenés à considérer que toute sur-réglementation ("germanisation») risque de mener à un déséquilibre entre la distribution spatiale du problème et la distribution de l'intervention publique, alors même qu'on cherche à combattre ce déséquilibre; en effet, le renforcement du programme national a tendance à "schématiser" la mise en œuvre, ce qui peut "rendre aveugle» l'autorité publique. Etant donné qu'en France, la politique de mise en œuvre des régions est très liée au niveau de programmation nationale, et qu'il n'y a pas une nécessité d'harmoniser les politiques régionales de mise en œuvre par le biais du programme national (comme c'est le cas pour un Etat fédéral comme la RFA, ou fortement régionalisé comme I'Italie, pour assurer "l'égalité de traitement»), un tel renforcement est inoppor. tun.

De plus, en France, l'existence du corps des mines, sa cohérence et son unité de doctrine peuvent être considérées comme l'équivalent des réglementations des autres pays. Un renforcement des éléments du programme national risquerait de bloquer la capacité innovatrice des DII, telle qu'elle s'est manifestée durant les années 1970. Tant que I'action des DII restera efficace et efficiente, il n'y aura pas besoin de plus d'éléments substantiels dans le programme national, autres que ceux mentionnés.

C'est l'analyse des politiques des régions d'lle de France et du Nord Pas-deCalais qui nous permet de conclure que l'équilibre entre l'intervention publique et la pression du problème peut être atteint non pas, par une exclusion de toutes intéractions («technicisation complète»), mais au contraire par une gestion de ces intéractions par les DII. Si l'administration chargée de la mise en cuvre a une capacité technique et de négociation suffisante pour la rendre indépendante du réseau des intéractions, elle peut utiliser les pressions politiques des collectivités locales, du gouvernement central et même des associations régionales ou locales d'industriels. Ce sont les DII françaises qui paraissent avoir développé le plus cette capacité de gestion : dans les autres pays étudiés, cette capacité à gérer le problème s'est avérée très faible, car les fonctionnaires sont soit des administrateurs, soit des techniciens, mais rarement des "gestionnaires». La création d'un réseau d'intéraction équilibré nous semble la meilleure stratégie à adopter pour garantir une distribution de l'intervention publique adaptée à la 
distribution du problème. Certes, la "germanisation» des politiques publiques en impose aux visiteurs étrangers, mais son application dans le concret mène plus à la production d'actes administratifs compliqués qu'à une plus grande efficacité.

- La généralisation de l'aspect distributif : sans présenter une analyse détaillée (16), la recherche menée montre que l'étude des effets distributifs des politiques publiques ne doit pas être limitée à l'analyse de politiques distributives ou redistributives dans le sens de LOWI (17). Les interventions régulatrices, comme les activités de contrôle, de sanction, de persécution ou d'interdiction, créatrices de biens publics (comme la Sécurité, la Santé ou la Qualité de Vie) peuvent être distribuées par la fonction publique tout comme l'argent. La comparaison des stratégies dans le domaine de la politique de l'environnement montre que l'on peut améliorer la qualité de l'environnement dans certains espaces, soit par l'application d'instruments régulateurs aux émetteurs principaux de ces espaces, soit par l'attribution de subventions publiques à ces émetteurs. On peut créer des modèles de distribution inégalitaire par l'attribution de subventions qui favorisent certains espaces par rapport à d'autres; mais on peut atteindre le même résultat par une concentration de l'attention administrative et de l'intervention publique sur un espace, au détriment des autres. Contrairement à ce que l'on pense généralement ( $y$ compris les politiciens et les politistes), la généralisation d'une législation régulatrice ne garantit pas son application avec la même inten. sité "sur l'ensemble du territoire» d'un pays. La distribution spatiale de l'intervention publique, pour les programmes ayant une répercussion spatiale, est parmi les plus intéressantes à étudier.

- Le facteur politique local : étant donné l'importance des effets distributifs spatiaux de toutes activités régionales ou départementales de mise en œuvre de politiques publiques ayant une répercussion spatiale importante, l'analyse des modèles explicatifs de la distribution spatiale de l'intervention publique doit se concentrer vers le rôle politique des collectivités locales concernées lors des négociations avec les administrations de mise en œuvre. Cela est surtout vrai pour les villes grandes et moyennes, dans lesquelles les notables politiques peuvent influencer la politique de mise en œuvre par des pressions directes sur l'administration régionale, ou indirectes en intervenant auprès des ministères nationaux (surtout en France), qui peuvent à leur tour avoir une influence sur le plan régional ou départemental. Dans le cas de la France, comme dans le cas d'autres pays, on peut penser que ces pressions sont responsables de la distribution inégalitaire de l'intervention administrative, qui favorise des collectivités locales au détriment d'autres (18). Notre recherche a montré, qu'en France, la capacité d'une collectivité locale à attirer la bienveillance des politiques de mise en cuvre régionale ne dépend pas uniquement de la couleur politi- 
que de l'administration locale (19), mais également d'autres facteurs politiques comme la capacité d'attirer l'attention des médias pour augmenter la portée d'un cas local, l'existence de mouvements sociaux opposés ou favorables à la mise en œuvre d'une certaine politique, l'existence d'administrés d'importance nationale (comme les industries à Fos ou à Dunkerque) ou les stratégies électorales du gouvernement national (20). En France comme en Italie, le facteur politique local joue un rôle important (21), selon notre recherche dans le domaine de la pollution de l'air ; nous avons découvert deux types d'influence : les "freineurs locaux" ("local breakman») : c'est le cas de la ville de Marseille, qui a pu, grâce à son poids politique important, éviter quasiment toute intervention publique systématique sur son territoire pendant environ 10 ans (22). Les «accélérateurs locaux" ( «local mobilizers») : c'est le cas en France de la Communauté Urbaine lilloise et de Fos-Etang de Berre-Martigues. Ces deux exemples montrent que les "accélérateurs locaux" peuvent accélérer l'intervention publique dans les espaces où il y a une forte pression de problème, comme dans les espaces où la pression du problème ne justifie pas une telle intervention (23).

En conclusion, nous soulignerons que l'austérité fiscale des Etats Providences occidentaux va accentuer le conflit de la distribution de l'intervention publique, dans la mise en œuvre des politiques distributives comme des politiques régulatrices. Plus on découvrira la distribution inégalitaire des problèmes sur le plan spatial et ses répercussions sur les politiques publiques (moins évidentes durant les périodes de croissance fiscale), plus le rôle joué par le facteur politique locale sera important pour la politique de mise en œuvre, quelle que soit l'administration de mise en œurre choisie (nationale, régionale ou locale). Ce n'est pas en période d'abondance, mais en période de crise que ces inégalités spatiales apparaissent ; et il incombe aux politiciens, aux administrations publiques, mais aussi aux politistes d'éviter le pire. 


\section{NOTES}

(1) L'Etat de l'Environnement, 1983 ; Ministère de l'Environnement, DPP ; Documentation Française ; pp. 119 et 120. Graphique 2321.

(2) P. KNOEPFEL, H. WEIDNER (1982) : «Formulation and Implementation of Air Quality Control Programmes : Patterns of Interest Consideration». Policy and Politics, Vol. 10, $\mathrm{n}^{\circ} 1$, pp 96 ss.

(3) Voir pour la RFA la TA-Luft (Technische Anleitung zur Reinhaltung der Luft $=1$. Verwaltungsvorschrift zum Bundesimmissionsschutzgesetz) du 28/8/1974. Pour la Suisse : la nouvelle ordonnance d'application de l'article 7 de la Loi Fédérale pour la Protection de l'Environnement concernant les grandes unités de combustion, qui prévoit l'installation d'un équipement de désulfuration des fumées pour ces unités (projet d'une Ordonnance concernant la Protection contre la Pollution Atmosphérique de mai 1984, annexes 2 et 3 ).

(4) En Suisse, RFA et aux Pays-Bas, la mise en œuvre de la politique de l'Environnement incombe aux autorités ordinaires de l'administration publique, soumises au contrôle politique normal, parfois exercé de façon très ouverte. (Suisse : administrations cantonales ; RFA : administrations des Landers et des Districts administratifs, à l'exception de quelques Landers, qui ont des administrations techniques spéciales comparables aux DII ; Pays-Bas : I'administration ordinaire des Provinces). Voir aperçu dans : P. KNOEPFEL, H. WEIDNER (1980) : Handbuch der $\mathrm{SO}_{2}$ Luftreinhaltepolitik, vol. 1 : Vergleichende Analyse, Berlin (E. Schmidt), pp. 102 ss.

(5) La qualité reconnue aux ingénieurs des mines, le sérieux de leur formation (connue également par les industries dans lesquelles de nombreux ingénieurs du corps "pantouflent»), font des DII un partenaire majeur aux yeux des industriels.

(6) Voir E. FRIEDBERG et D. DESJOUX (1973) : "Fonctions de I'Etat et Rôle des Grands Corps : le Cas du Corps des Mines» ; Annuaire de la Fonction Publique, Paris.

(7) Dans : Ministère de l'Environnement (1978): Les pratiques réglementaires en Matière de Pollutions et Nuisances Industrielles en France, DPP, ronéo. 
(8) Voir H. de MAGALHAES, C. LARRUE, R. DARBERA (1982) : «Politique Nationale et Mise en Oeuvre du Contrôle de la pollution atmosphérique par le $\mathrm{SO}_{2}$ en France», Rapport final de recherche, IUP/IIES, Paris/ Berlin, (polycopié).

(9) Voir également l'analyse intéressante de H.C. BUGGE (1976) : La Pollution Industrielle : Problèmes juridiques et Administratifs, PUF, pp 268284.

(10) Voir le livre toujours actuel de S. BARRETT, C. FUDGE (eds.) (1981): Policy and Action - Essays on the Implementation of Public Policy, London \& New York. Et les deux ouvrages produits par un cercle de chercheurs allemands : R. MAYNTZ (Hrsg.) (1980) : Implementation politischer Programme, Empirische Forschungsberichte, Königstein Ts (Athenäum), ainsi que R. MAYNTZ (Hrsg.) (1983) : Implementation politischer Programme II, Ansätze zur Theoriebildung, Opladen (Westdeutscher Verlag).

(11) La valeur limite en RFA à fixti selon la loi sur la protection de la pollution atmosphérique (Bundesimmissionsschutzgesetz art. 10 al. 1 et art. 49 al. 2) et qui a été fixée dans la Nordrhein-Westfalia (1978), dans la Sarre (1976), à Hessen (1976-77), dans le Rheinland-pfalz (1976), au Bade Wurttemberg (1977) et à Berlin (1977) est de $800 \mu \mathrm{g} / \mathrm{m} 3 \mathrm{de} \mathrm{SO}_{2}$. Le dépassement de cette valeur déclenche le premier niveau d'alerte. Voir en particulier : V. PRITTWITZ : «Vorausschauende Smogbekämpfung. Materialien und Ueberlegungen zum Stand der Luftreinhaltepolitik in Ballungsräumen der Bundesrepublik Deutschland», IIUG dp (Berlin) $n^{\circ} 81-5$.

(12) La plupart des conclusions présentées ici sont tirées du rapport final à la Deutsche Forschungsgemeinschaft, Berlin (1984), P. KNOEPFEL et H. WEIDNER (en allemand).

(13) Les 14 régions inclues dans la recherche sont : Berlin, Bavière, Nordrhein-Westfalia pour la RFA ; Greater-London et South Yorkshire pour la Grande-Bretagne ; Provence Côte d'Azur, lle de France et Nord Pasde-Calais pour la France; Piémont, Lombardie et Emilie-Romagna pour I'Italie ; et Nord-Holland, Rijmond et Gelderland pour les Pays-Bas. A l'intérieur de ces 14 régions, nous avons analysé 30 unités locales plus précisément.

(14) Le débat sur le dépérissement des forêts, mené avec une grande intensité en Suisse et en RFA, semble avoir démarré récemment en France. 
(15) Voir pour la RFA : P. KNOEPFEL, H. WEIDNER (1983) : «Die Durchsetzbarkeit planerischer Ziele auf dem Gebiet der Luftreinhaltung aus der Sicht der Politikwissenschaft. Ergebnisse aus einer internationalen Ver. gleichsstudie», Zeitschrift für Umweltpolitik / Revue de la Politique de l'Environnement $n^{\circ}$ 2/83 p 87-115 Frankfurt (Deutscher Fachverlag $\mathrm{GmbH})$ : VDI-Berichte $n^{\circ} 477$ (Reinhaltung der Luft in grossen Städten), Düsseldorf 1983, p 191-208. Voir pour I'Italie B. DENTE, P. KNOEP. FEL, R. LEWANSKI, S. MANNOZZI, S. TOZZI (1984) : Il controllo dell'inquinamento atmosferico in Italia : Analisi di una politica regolativa contro l'inquinamento atmosferico, Roma (officina), ou le résumé par R. DENTE et R. LEWANSKI (1983) : "Implementing Air Pollution Control Policies in Italy : The Importance of the Political and Administrative Structure», in P. DOWNING, K. HANF (eds.) : International Comparisons in Implementing Pollution Laws, Boston, Dordrecht (KluwerNijhoff), p. 107-128.

(16) Voir pour une analyse plus détaillée : P. KNOEPFEL (1984) : "Distributional Issues in Regulatory Policy Implementation - The Case of Air Quality Control Policies», Contribution to the International Symposium on "Distributional Conflicts in Environmental-Ressource Policy" at the IIES in Berlin on 26-27 March 1984, publication prévue dans WATTS N., SCHNAIBERG A., ZIMMERMANN K. (eds.) : Distributional Conflicts in Environmental Policy.

(17) Voir : LOWI T.J. (1972) : «Four systems of policy, politics and choice, Public Administration Review, 32 (July/August) ; pp. 298-310.

(18) C'est exactement la question commune des recherches du projet «Allocation of Social Benefits", mené à l'initiative de D.E. ASHFORD (University of Pittsburgh), auquel participent des équipes de recherche des Etats-Unis, de I'Angleterre, de la France, de la Norvège, de l'Italie, du Japon, de I'Allemagne Fédérale, d'Israël, du Danemark et de la Suisse.

(19) Cette variable est favorisée dans le concept analytique pour la recherche "Allocation of Social Benefits" (cf note 18). Dans le cas de la recherche italienne (direction B. DENTE), ou de la Suisse (direction P. KNOEP. FEL), cette variable s'est avérée moins importante que dans le cas d'au. tres pays.

(20) Cette question est examinée dans le cadre du projet de recherche français : "Analyse des relations entre l'Etat et les Collectivités Locales dans le cadre de la définition et de la mise en cuure des politiques sociales", dirigé par F. CHAZEL, J.C. THOENIG et J. DUMAS et réalisé par P. DURAN à I'Université de Bordeaux. 
(21) Voir pour le rôle important du facteur politique local pour l'Italie : B. DENTE, R. LEWANSKI : op. cit. (note $n^{\circ} 15$ ). pp 113 ss.

(22) L'équivalent en Italie a pu être trouvé dans la commune de Piacenza et de Turin. Voir : P. KNOEPFEL : Distributional Issues in Regulatory Policy Implementation (note $n^{\circ} 16$ ), p $13 \mathrm{~s}$.

(23) L'équivalent en Italie a pu être trouvé à Chivasso, commune qui a été capable d'attirer l'intervention publique au détriment des villes de Turin et de Moncalieri, localités beaucoup plus polluées que Chivasso. Voir P. KNOEPFEL, op. cit. note 16 et 22, p. 14. 